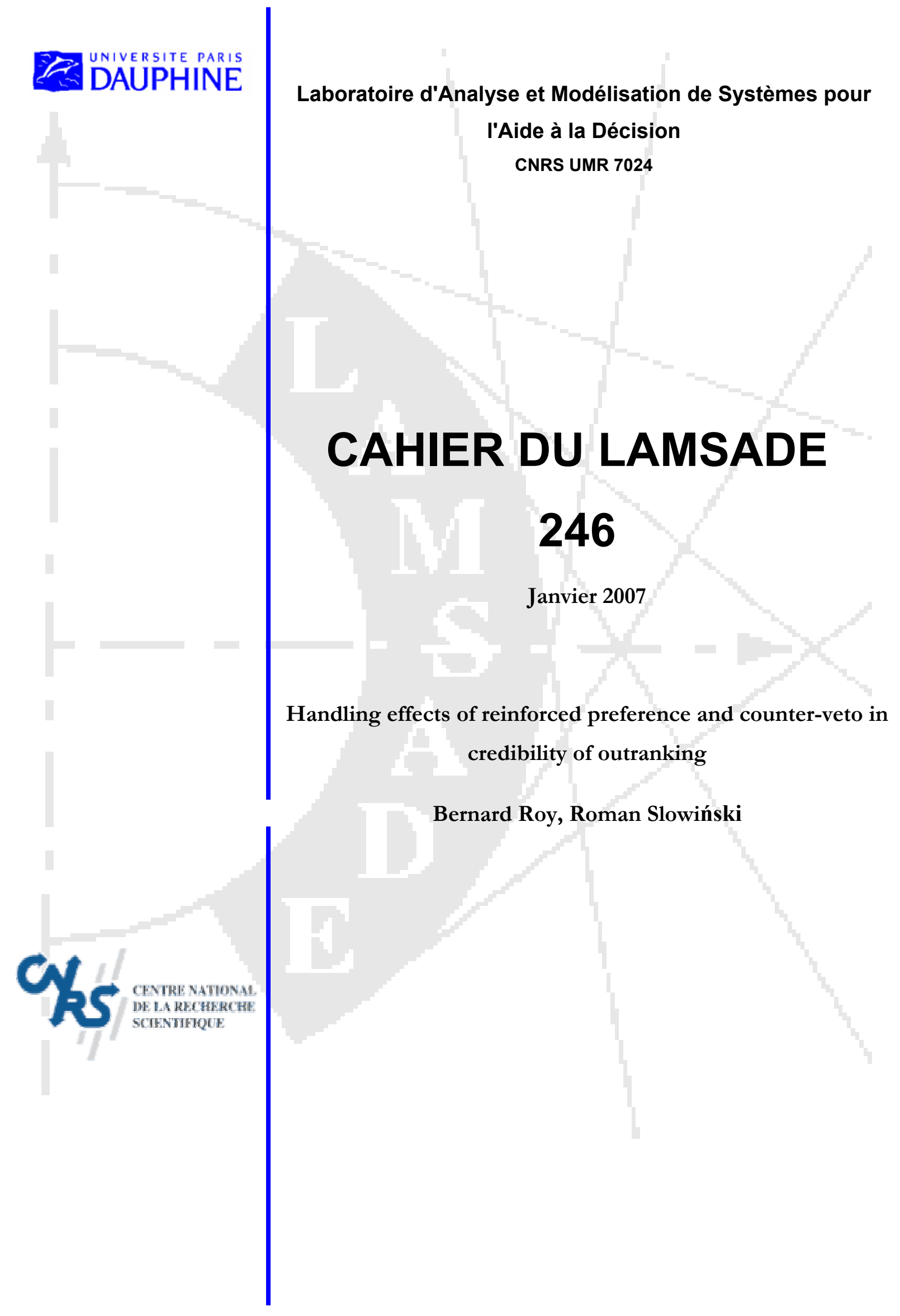




\title{
Handling effects of reinforced preference and counter-veto in credibility of outranking
}

\author{
Bernard Roy ${ }^{1}$, Roman Stowiński ${ }^{2}$ \\ ${ }^{1}$ LAMSADE, Paris-Dauphine University, 75775 Paris, France; \\ roy@lamsade.dauphine.fr \\ ${ }^{2}$ Institute of Computing Science, Poznań University of Technology, 60-965 Poznań, \\ and Institute for Systems Research, Polish Academy of Sciences, 01-447 Warsaw, Poland; ro- \\ man.slowinski@cs.put.poznan.pl
}

\begin{abstract}
The aim of this paper is to generalize the way of computing the credibility of outranking in a multiple criteria aggregation procedure, in view of taking into account two new effects called reinforced preference and counter-veto. These effects concern only those criteria for which, as soon as action $a$ is "judged very strongly preferred" to action $b$, one wishes that the credibility of outranking of $a$ over $b$ is greater than that for the case where (all things equal elsewhere) the preference is not "judged very strong". To achieve this goal, we propose two complementary ways. The first one involves a reinforced preference threshold which affects the concordance degree, and the second one involves a counter-veto threshold which affects the insertion of discordance degree in the calculation of the credibility of outranking. The introduction of these two new effects remains compatible with the handling of purely ordinal preference scales. The resulting new index of the credibility of outranking can be used, in particular, in ELECTRE methods.
\end{abstract}

Keywords: Decision support, Multiple criteria analysis, Outranking, Concordance, Discordance. 


\title{
Prise en compte de préférences renforcées et contre veto dans la crédibilité d'un surclassement
}

\begin{abstract}
Résumé
L'objet de cet article est d'étendre le mode de calcul de la crédibilité d'un surclassement dans une procédure d'agrégation multicritère afin de pouvoir prendre en compte deux nouveaux effets appelés préférences renforcées et contre veto. Ces effets ne concernent que les critères à propos desquels, dès lors qu'une action $a$ est « jugée très fortement préférée » à une action $b$, on souhaite que la crédibilité du surclassement de $a$ vis-à-vis de $b$ soit plus élevée que ce qu'elle serait (toutes choses égales par ailleurs) si la préférence n'était pas "jugée très forte ». Deux possibilités complémentaires sont proposées pour atteindre ce but. L'une repose sur l'introduction d'un seuil de préférence renforcée qui affecte le degré de concordance et l'autre sur celle d'un seuil de contre veto qui affecte le degré de discordance.

L'introduction de ces deux nouveaux effets reste compatible avec la prise en compte d'échelles de préférence purement ordinales. La nouvelle définition de l'indice de crédibilité du surclassement qui en résulte peut, en particulier, être substituée à l'ancienne dans les méthodes ELECTRE, sans apporter d'autres modifications.
\end{abstract}

Mots clés : Aide multicritère à la décision ; Surclassement ; Concordance ; Discordance. 


\section{Motivations}

Suppose that a consistent family of criteria $F=\left\{g_{1}, g_{2}, \ldots, g_{n}\right\}$ has been build in view of comparing actions from a finite set $A=\{a, b, c, \ldots\}$. Each criterion $g_{j}, j=1, \ldots, n$, may be associated with indifference and/or preference thresholds, denoted as $q_{j}$ and $p_{j}$, respectively $[1,8,9]$. We assume, without loss of generality, that the preference increases with the performance level $g_{j}(a), a \in A$. We are considering comparisons of actions which result in an outranking relation $S$ for all ordered pairs of actions $(a, b) \in A$, such that $a S b$ means " $a$ is at least as good as $b$ ". The assertion $a S b$ involves two concepts known as concordance and discordance. The outranking relation $a S b$ is characterized by the credibility index $\sigma(a, b)[1]$. The way of calculating $\sigma(a, b)$ will be reminded in Section 2.

The credibility index involves preference scales which are purely ordinal. For this reason, as soon as on criterion $g_{j}$, the difference of performances $g_{j}(a)-g_{j}(b)$ becomes greater than the preference threshold, the value of this difference does not influence the credibility of outranking of action $a$ over action $b$. If one would judge that a very large value of this difference gets the meaning of "very strong preference", then one could wish to take this judgment into account in the definition of the credibility of outranking of $a$ over $b$. To satisfy such a wish, we are proposing two complementary ways:

- the first one involves a new threshold called reinforced preference threshold: it corresponds to the value of the difference of performances $g_{j}(a)-g_{j}(b)$ which is "judged meaningful" for considering criterion $g_{j}$ as more important in the concordant coalition (by increasing its weight), comparing to the situation where (all things equal elsewhere) the difference of performances is smaller than this threshold (however, not smaller than the preference threshold);

- the second one involves another threshold called counter-veto threshold (it is not necessarily equal to the previous one, as it has a different meaning and it plays a different role): it corresponds to the value of the difference of performances $g_{j}(a)$ - $g_{j}(b)$ which is "judged meaningful" for weakening the mechanism of veto against the credibility of outranking (from the side of discordant criteria), comparing to the situation where (all things equal elsewhere) the difference of performances is smaller than this threshold (however, not smaller than the preference threshold).

These two ways are presented in Sections 3 and 4, respectively. In the last Section, we comment the possible use of the new credibility index, in particular, within ELECTRE methods.

\section{Credibility of outranking $-\mathbf{a}$ brief reminder}

Consider an ordered pair of actions $(a, b) \in A$ and a hypothesis of the outranking $a S b$. The credibility of outranking is usually defined by the credibility index $\sigma(a, b)$, as follows:

$$
\sigma(a, b)=C(a, b) \times \Delta(a, b),
$$

where $C(a, b)$ is a concordance degree and $\Delta(a, b)$ is a component taking into account discordance degrees on particular criteria. For the sake of simplicity, in all formulae that follow, we suppose that all thresholds are constant and that criteria are identified by their indices. 
Let us denote by $k_{j}$ the weight assigned to criterion $g_{j}, j=1, \ldots, n$; it represents a relative importance of criterion $g_{j}$ within family $F$.

The concordance degree $C(a, b)$ takes account of the strength of the coalition of criteria being in favor of the assertion " $a$ is at least as good as $b$ ", i.e. in favor of $a S b$. This coalition is composed of two subsets of criteria:

- $\quad \mathcal{C}(a S b)$ - composed of criteria being clearly in favor of $a S b$, i.e. such that $g_{j}(a) \geq$ $g_{j}(b)-q_{j}$,

- $\boldsymbol{e}(b Q a)$ - composed of criteria that do not oppose to $a S b$, while being in an ambiguous position with respect to this assertion; these are those criteria for which a weak preference relation $b Q a$ holds; i.e. such that $g_{j}(b)-p_{j} \leq g_{j}(a)<g_{j}(b)-q_{j}$,

Consequently, the concordance degree is defined as

$$
C(a, b)=\frac{\sum_{j \in \mathcal{e}(a S b)} k_{j}+\sum_{j \in \mathcal{e}(b Q a)} \varphi_{j} k_{j}}{\sum_{j=1}^{n} k_{j}}, \text { where } \varphi_{j}=\frac{g_{j}(a)-\left(g_{j}(b)-p_{j}\right)}{p_{j}-q_{j}} .
$$

$\varphi_{j} \in[0,1]$ is a coefficient that decreases the contribution of criterion $g_{j}$ to the concordance degree as far as the ambiguity increases.

Remark that $C(a, b) \in[0,1]$, where $C(a, b)=0$ if $g_{j}(a) \leq g_{j}(b)-p_{j}, j=1, \ldots, n$ ( $b$ is strictly preferred to $a$ on all criteria), and $C(a, b)=1$ if $g_{j}(a) \geq g_{j}(b)-q_{j}, j=1, \ldots, n$ ( $a$ outranks $b$ on all criteria).

Let us consider now those criteria for which a finite veto threshold $v_{j}$ has been specified. Each one of these criteria is associated with a degree of discordance defined as

$$
d_{j}(a, b)=\left\{\begin{array}{ccc}
1, & \text { if } & g_{j}(a) \leq g_{j}(b)-v_{j} \\
{\left[\left(g_{j}(b)-v_{j}{ }^{\prime}\right)-g_{j}(a)\right] /\left(v_{j}-v_{j}{ }^{\prime}\right)} & \text { if } & g_{j}(b)-v_{j}<g_{j}(a) \leq g_{j}(b)-v_{j}{ }^{\prime}, \\
0 & \text { if } & g_{j}(a)>g_{j}(b)-v_{j}{ }^{\prime}
\end{array}\right.
$$

where $v_{j}{ }^{\prime}$ is called pre-veto threshold, $v_{j}>v_{j}{ }^{\prime} \geq p_{j}(j=1, \ldots, n)$. Originally [7], $v_{j}{ }^{\prime}=p_{j}$, however, in some situations it might be desirable to keep $d_{j}(a, b)=0$ when $g_{j}(a)<g_{j}(b)-p_{j}$, which would make $v_{j}{ }^{\prime}>p_{j}$. Such situations were pointed out as well in methodological studies $[3,5,9,11]$, as in practical applications [6].

There are two ways of defining $\Delta(a, b)$, either as:

$$
\Delta(a, b)=\prod_{j \in J(a, b)} \frac{1-d_{j}(a, b)}{1-C(a, b)},
$$

where $j \in J(a, b)$ if and only if $d_{j}(a, b) \geq C(a, b)$, which is the original formula, or as

$$
\Delta(a, b)=\prod_{j \in F}\left(1-d_{j}(a, b)\right) .
$$


When using formula (2.5), one might wish to avoid situation where veto intervenes prematurely without sufficient justification; then it is recommended to set $v_{j}{ }^{\prime}>p_{j}$ for all those criteria for which $v_{j} \neq \infty$.

It follows from the above formulae that $\sigma(a, b) \in[0,1]$.

\section{Handling of preference reinforcement thresholds}

\subsection{Principles and requirements}

The goal is to give to certain criteria $g_{j}, j \in F$, the possibility of increasing their relative importance in the definition of concordance degree $C(a, b)$ when the difference $g_{j}(a)-$ $g_{j}(b)$ exceeds a given value called reinforced preference threshold. The handling of preference reinforcement thresholds should satisfy the following requirements:

i) all things equal elsewhere, if for any criterion $g_{j}, j \in F$, the reinforced preference threshold is crossed, then the concordance degree $C(a, b)$ cannot decrease,

ii) $C(a, b)$ should remain a monotone non-decreasing function with respect to differences $g_{j}(a)-g_{j}(b)$ for each $j \in F$,

iii) $C(a, b)$ should remain equal to 1 if and only if $a$ outranks $b$ on all criteria.

\subsection{Proposed formula}

Let $r p_{j}$ denote the reinforced preference threshold for criterion $g_{j}$. When this threshold is crossed, the importance coefficient $k_{j}$ in formula (2.2) should be replaced by $\omega_{j} k_{j}$, where $\omega_{j}>1$ is called reinforcement factor. Let $\mathcal{C}(a R P b)$ denote the set of criteria for which $g_{j}(a)>g_{j}(b)+r p_{j}$. The concordance degree in then defined as

$$
C(a, b)=\frac{\sum_{j \in \mathcal{e}(a R P b)} \omega_{j} k_{j}+\sum_{j \in \mathcal{e}(a S b)-\boldsymbol{e}(a R P b)} k_{j}+\sum_{j \in \mathcal{e}(b Q a)} \varphi_{j} k_{j}}{\sum_{j \in \mathcal{e}(a R P b)} \omega_{j} k_{j}+\sum_{j \in F-\boldsymbol{e}(a R P b)} k_{j}} .
$$

\subsection{Proofs of requirements}

Proof of i). Let $(a, b) \in A$ such that $C(a, b)=N / D$. Remark that $N \leq D$. Consider criterion $g_{j}$ such that $g_{j}(b)-q_{j} \leq g_{j}(a) \leq g_{j}(b)+r p_{j}$. Suppose that $g_{j}(a)$ has grown such that $g_{j}(a)>$ $g_{j}(b)+r p_{j}$. Then the concordance degree becomes

$$
\begin{gathered}
C^{\prime}(a, b)=\frac{N+\left(\omega_{j}-1\right) k_{j}}{D+\left(\omega_{j}-1\right) k_{j}} . \\
C^{\prime}(a, b) \geq C(a, b) \Leftrightarrow \frac{N+\left(\omega_{j}-1\right) k_{j}}{D+\left(\omega_{j}-1\right) k_{j}} \geq \frac{N}{D} \Leftrightarrow D\left(\omega_{j}-1\right) k_{j} \geq N\left(\omega_{j}-1\right) k_{j}, \text { which is true }
\end{gathered}
$$
because $N \leq D$.

Proof of ii). According to i), requirement ii) holds when $g_{j}(a)-g_{j}(b)$ on criterion $g_{j}, j \in F$, is crossing $r p_{j}$. In all other cases, the increase of $g_{j}(a)-g_{j}(b)$ keeps the denominator of (3.1) unchanged. Since in such cases nominator is a non-decreasing function of each $g_{j}(a)-g_{j}(b)$, the requirement is satisfied. 
Proof of iii). If $a$ outranks $b$ on all criteria, then $\boldsymbol{e}(b Q a)=\varnothing$ and $\boldsymbol{e}(a S b)=F$; then, independently of $\mathcal{C}(a R P b)$, the nominator of (3.1) becomes equal to the denominator. Suppose now that there exists at least one criterion $g_{j}, j \in F$, for which $g_{j}(a)<g_{j}(b)-q_{j}$. In this case, $j$ does not belong neither to $\mathcal{C}(a R P b)$ nor to $\mathcal{C}(a S b)$, however, it can belong to $\boldsymbol{e}(b Q a)$. Even so, the nominator of (3.1) is strictly smaller that the denominator, thus $C(a, b)<1$.

\section{Handling of counter-veto thresholds}

\subsection{Principles and requirements}

The goal is to give to certain criteria $g_{j}, j \in F$, the possibility of decreasing the veto effect on discordant criteria when the difference $g_{j}(a)-g_{j}(b)$ exceeds a given value called counter-veto threshold. By definition,

case a) if no veto effect intervenes on any criterion $(\Delta(a, b)=1$, see Section 2$)$, then the counter-veto of $g_{j}$ is inactive,

case b) if veto effects intervene on some criteria $(\Delta(a, b)<1)$, then the counter-veto of $g_{j}$ is weakening these effects.

The handling of counter-veto thresholds should satisfy the following requirements:

i) all things equal elsewhere, if the number of criteria $g_{j}, j \in F$, for which the counter-veto threshold is crossed, increases from $k$ to $k+1$, then the credibility of outranking $\sigma(a, b)$ cannot decrease, i.e. $\sigma(a, b)$ is a monotone, non-decreasing function of $k$,

ii) if there exists a discordant criterion $g_{i}, i \in F$, which does not produce a veto effect, then, all things equal elsewhere, withdrawing this criterion from family $F$ should keep increasing the credibility of outranking $\sigma(a, b)$, whatever number $k$ of criteria for which the counter-veto threshold has been crossed,

iii) if for a discordant criterion $g_{i}, i \in F$, the discordance degree $d_{i}(a, b)$ increases, then, all things equal elsewhere, the credibility of outranking $\sigma(a, b)$ should remain non-increasing, whatever the number $k$ of criteria for which the counterveto threshold has been crossed, i.e. $\sigma(a, b)$ is a monotone, non-increasing function of $d_{i}(a, b)$.

\subsection{Proposed formula}

Let $c v_{j}$ denote the counter-veto for criterion $g_{j}$ and $k$ the number of criteria for which the counter-veto threshold has been crossed. We propose to define the index of the credibility of outranking $\sigma(a, b)$ as

$$
\sigma(a, b)=C(a, b) \times[\Delta(a, b)]^{(1-k / n)} .
$$

In this formula, $C(a, b)$ can be defined by either (2.2) or (3.1), and $\Delta(a, b)$ by either (2.4) or (2.5). This formula satisfies both properties expressed as case a) and case b) in the definition. As to case a), for $\Delta(a, b)=1,[\Delta(a, b)]^{(1-k / n)}=1$ whatever $k$. As to case b), $\Delta(a, b) \leq[\Delta(a, b)]^{(1-k / n)}$, whatever $k$, since $\Delta(a, b)<1$. 


\subsection{Proofs of requirements}

Proof of i). Since $\Delta(a, b) \leq 1,[\Delta(a, b)]^{(1-k / n)}$ is a monotone, non-decreasing function of $k$.

Proof of ii). We consider two cases related to the definition of $\Delta(a, b)$ :

- if $\Delta(a, b)$ is defined by (2.4), then discordant criterion $g_{i}, i \in F$, has to verify $0 \leq d_{i}(a, b) \leq C(a, b)$,

- if $\Delta(a, b)$ is defined by (2.5), then discordant criterion $g_{i}, i \in F$, has to verify $d_{i}(a, b)=0$.

In each of these cases, withdrawing $g_{i}$ from family $F$ keeps $\Delta(a, b)$ unchanged. This withdrawal changes $n$ to $n-1$, thus the exponent decreases from $1-k / n$ to $1-k /(n-1)$, which makes $[\Delta(a, b)]^{(1-k / n)} \leq[\Delta(a, b)]^{(1-k /(n-1))}$. On the other hand, withdrawing $g_{i}$ from family $F$ increases $C(a, b)$ because its denominator decreases by $k_{i}$ while its nominator remains unchanged. Consequently, the credibility of outranking $\sigma(a, b)$ is increasing.

Proof of iii). Independently of the definition of $\Delta(a, b)$, the increase of any $d_{i}(a, b)$ cannot increase $\Delta(a, b)$ and, consequently, $[\Delta(a, b)]^{(1-k / n)}$ cannot increase.

\section{Conclusions}

The aim of this paper was to handle the effect of reinforced preference defined in 3.1 and the effect of counter-veto defined in 4.1. We have shown that formula (4.1) takes into account these effects in accordance with requirements expressed in 3.2 and 3.3.

Let us remark that these effects concern only those criteria for which, as soon as action $a$ is "judged very strongly preferred" to action $b$, one wishes that the credibility of outranking of $a$ over $b$ is greater than the one for the case where (all things equal elsewhere) the preference is not "judged very strong".

In all formulae considered in the paper, all thresholds have been supposed constant for the sake of simplicity only, but one can easily re-write these formulae with variable thresholds.

For any criterion $g_{j}, j \in F$, the two thresholds $r p_{j}$ and $c v_{j}$ can be chosen equal, and, moreover, one may wish to consider only one of the two effects; deleting an effect means giving to the corresponding threshold an infinite or very large value. Consequently, no order relation is imposed between these two thresholds.

The new formula (4.1) for the index of the credibility of outranking can be substituted for similar formulae used in original versions of ELECTRE III and ELECTRE TRI [1].

The assignment of values to the new thresholds $r p_{j}$ and $c v_{j}$ can be done in a constructive way of thinking about the model of decision problem at hand. One can use for this some protocols of inquiry similar to those proposed for assigning appropriate values to indifference and preference thresholds [10], or to the weights [2]. These protocols involve few easy questions which do not require from the addressee to speculate about completely unrealistic situations. Another way could be to proceed via disaggregationaggregation approach, so as to get thresholds $r p_{j}$ and $c v_{j}$ as compatible as possible with some exemplary pairwise comparisons of few real actions [4]. 
The way of introducing the two new effects is consistent with the handling of purely ordinal preference scales. Each of the two new thresholds is like a frontier representing a qualifier without any reference to a notion of quantity. The weights remain intrinsic weights, and do not become substitution rates, the indifference and preference thresholds play exactly the same role as before.

The new formula could also be used outside ELECTRE methods, for example, as similarity or closeness index $[11,12,13]$, or as a filtering operator [5].

\section{References}

[1] Figueira, J., Mousseau, V. and Roy, B.: ELECTRE methods. Chapter 4 [in]: J. Figueira, S. Greco and M. Ehrgott (eds.), Multiple Criteria Decision Analysis: State of the Art Surveys, Springer-Verlag, New York, 2005, pp. 133-162.

[2] Figueira, J. and Roy, B.: Determining the weights of criteria in the ELECTRE type methods with a revised Simos' procedure. European Journal of Operational Research, 139 (2002) 317-326.

[3] Mousseau, V. and Dias, L.: Valued outranking relations in ELECTRE providing manageable disaggregation procedures. European Journal of Operational Research, 156 (2004) 467-482.

[4] Mousseau, V. and Słowiński, R.: Inferring an ELECTRE TRI model from assignment examples. Journal of Global Optimization, 12 (1998) 157-174.

[5] Perny, P.: Multicriteria filtering methods based on concordance/non-discordance principles. Annals of Operations Research, 80 (1998) 137-167.

[6] Pictet, J.: personal communication 2005.

[7] Roy, B.: The outranking approach and the foundations of ELECTRE methods. Theory and Decision, 31 (1991) 49-73.

[8] Roy, B.: Multicriteria Methodology for Decision Aiding. Kluwer Academic Publishers, Dordrecht, 1996.

[9] Roy, B.: Paradigms and challenges. Chapter 1 [in]: J. Figueira, S. Greco and M. Ehrgott (eds.), Multiple Criteria Decision Analysis: State of the Art Surveys, Springer Science + Business Media Inc., New York, 2005, pp. 3-24.

[10] Roy, B., Bouyssou, D.: Aide multicritère à la décision: Méthodes et cas. Economica, Paris, 1993

[11] Słowiński, R. and Stefanowski, J.: Rough classification with valued closeness relation. [In]: E. Diday, Y. Lechevallier, M. Schrader, P. Bertrand, B. Burtschy (eds.), New Approaches in Classification and Data Analysis. Springer-Verlag, Berlin, 1994, pp. 482-489.

[12] Słowiński, R. and Vanderpooten, D.: Similarity relation as a basis for rough approximations. [In]: P.P. Wang (ed.): Advances in Machine Intelligence \& SoftComputing, Vol. IV, Duke University Press, Durham, NC, 1997, pp. 17-33. 
[13] Słowiński, R. and Vanderpooten, D.: A generalized definition of rough approximations based on similarity. IEEE Transactions on Data and Knowledge Engineering, 12 (2000) 331-336. 